\title{
Introducción. Rodolfo Lenz revisitado: un vector glotopolítico en un país en modernización
}

\author{
Juan Antonio Ennis ${ }^{1}$ \\ Universidad Nacional de La Plata / CONICET, Argentina \\ Darío Rojas ${ }^{2}$ \\ Universidad de Chile, Chile
}

\begin{abstract}
Resumen
El presente trabajo intenta contextualizar las contribuciones reunidas en este dosier, ofreciendo una caracterización de las líneas generales de la figura y obra del lingüista alemán Rodolfo Lenz, radicado en Chile desde 1890 hasta su muerte. Describimos su rol como el de un vector glotopolítico, esto es, una figura clave para la circulación y transformación de saberes y representaciones ideológicas sobre las lenguas de Chile y sus hablantes. Destacamos la fractura lingüísticoideológica que significó la llegada de Lenz al contexto cultural chileno
\end{abstract}

\footnotetext{
1Para correspondencia, dirigirse a: Juan Antonio Ennis (juanennis@conicet.gov.ar), Centro de Estudios de Teoría y Crítica Literaria, Instituto de Investigaciones en Humanidades y Ciencias Sociales (CONICET-Universidad Nacional de La Plata), calle 51 entre 124 y 125 (1925), Ensenada, Buenos Aires, Argentina.

2 Para correspondencia, dirigirse a: Darío Rojas (darioroj@uchile.cl), Universidad de Chile, Facultad de Filosofía y Humanidades, Departamento de Lingüística. Av. Capitán Ignacio Carrera Pinto 1025, piso 3, Ñunoa, Santiago, Chile.
} 
de fines del siglo XIX, dominado por el pensamiento normativista e hispanista inspirado en Andrés Bello. La condición polémica de las ideas de Lenz respecto de la ideología dominante explica en buena medida que su recuperación y revalorización, de la cual participan varios de los artículos de este volumen, sea relativamente reciente. Por otra parte, advertimos acerca del riesgo de levantar lecturas hagiográficas de Lenz sin considerar la complejidad de su contexto y el papel de sus obras en las políticas científicas, culturales y lingüísticas de su época.

Palabras clave: Rodolfo Lenz, glotopolítica, historiografía lingüística, castellano de Chile mapudungun.

\title{
RUDOLF LENZ REVISITED: A GLOTTOPOLITICAL VECTOR IN A MODERNIZING COUNTRY
}

\begin{abstract}
This paper aims to contextualize the articles gathered in this special issue, by means of a global characterization of the figure and work of the German linguist Rudolf Lenz, living in Chile from 1890 until his death. We describe his role as that of a glottopolitical vector, i.e. a key figure in the circulation and transformation of knowledge and ideological representations of the languages of Chile and their speakers. We highlight that his arrival to Chile caused a languageideological fracture, as he confronted the hegemony of linguistic normativism and hispanism, established on the basis of the influential works by Andrés Bello. His polemical stance towards the dominant ideology explains that the recovering and revaluation of his work is a relatively recent phenomenon, as some of the articles of this special issue show. On the other hand, we warn about the risk of raising hagiographic readings of Lenz without considering the complexity of his context and the role of his works in the scientific, cultural and linguistic policies of his time.
\end{abstract}

Keywords: Rudolf Lenz, glottopolitics, linguistic historiography. Chilean Spanish, mapudungun.

Recibido: 07/08/20

Aceptado: 28/09/20 


\section{LOS EFECTOS GLOTOPOLÍTICOS DE LA OBRA DE LENZ ${ }^{3}$}

El lingüista alemán Rudolf (Rodolfo) Lenz (Halle, 1863 - Santiago de Chile, 1938) fue una figura central en la conformación de varios campos disciplinares en el Chile de fines del siglo XIX y comienzos del XX. Entre estos sobresalen los estudios del lenguaje y las lenguas y la investigación sobre el folclore y las manifestaciones culturales llamadas "populares", tanto hispánicas como indígenas (cf. por ejemplo Mora y Vásquez 2018, para el caso de los estudios antropológicos), que Lenz colocó explícitamente en el centro de las tareas patrióticas que consideraba parte necesaria de la modernización de la nación chilena. Caracterizar a Lenz como un vector glotopolítico (la frase quiere aludir más o menos al modo en que se entienden los vectores en biología y epidemiología: un agente que sirve de medio de transmisión, capaz de portar y transmitir algo) implica destacar que fue un actor social de relevancia central en la conformación y reproducción de representaciones ideológicas sobre las lenguas y en la emergencia de políticas públicas acerca de ellas (así como acerca de sus manifestaciones culturales asociadas) en el Chile de fines del siglo XIX y comienzos del XX.

Formado intelectualmente en Alemania ${ }^{4}$, Lenz se radicó en Chile en enero de 1890. Allí llegó para formar parte del plan estatal de modernización de la producción y enseñanza de conocimientos, situado específicamente en el recién creado Instituto Pedagógico de la Universidad de Chile. En las últimas décadas del siglo XIX, la oligarquía chilena, enriquecida por la explotación del salitre, impulsó un acelerado proceso de modernización ligado a la expansión mundial del mercado capitalista (Subercaseaux 2011: 348), proceso que repercute directamente sobre las diversas instituciones y proyectos políticos y culturales de la época, entre ellas la educación. La modernización de la enseñanza se convirtió en la preocupación fundamental de intelectuales como Valentín Letelier, gracias a cuyos esfuerzos se decretó, en abril de 1889, la creación del Instituto Pedagógico, articulado sobre la necesidad de formar nuevos profesores a partir de criterios científicos y

\footnotetext{
Este trabajo se inscribe en el proyecto H850 "Filología, lingüística y archivo: aportes hacia una historia política de la lengua entre Europa y América Latina (mediados del siglo XIX-comienzos del siglo XX)", de la Universidad Nacional de La Plata, y en la investigación postdoctoral "En los bordes de la lengua: actitud e ideología lingüística en Andalucía y Chile (1850-1950)", financiada por el programa de Becas de Postdoctorado en el Extranjero - Becas Chile, convocatoria 2019, de ANID (ex CONICYT).

4 Véase Escudero (1963) para detalles de su biografía, así como el apartado 2 de esta introducción.
} 
sobre la contratación de docentes alemanes (de credenciales científicas reconocidas) para dicha institución. Dentro de estos, destacan Rodolfo Lenz, Federico Hanssen, Federico Johow, Augusto Tafelmacher, entre otros, quienes venían a impartir clases de humanidades -subdivididas en: historia y geografía, castellano y latín, inglés y alemán, griego y francés-y de ciencias -subdivididas en ciencias naturales y matemáticas- (Sanhueza 2010).

Desde esta posición privilegiada dentro de los campos científico y cultural chileno, respaldada y autorizada por un proyecto estatal de envergadura, Lenz se convirtió en vector de la circulación de saberes tanto entre Europa y Latinoamérica como entre las distintas naciones latinoamericanas. Pionero en la introducción en América Latina de la ciencia lingüística europea de fines del siglo XIX, y especialmente de los estudios sobre el contacto de lenguas, los procesos que protagoniza este intelectual tienen ramificaciones y repercusiones más allá del entorno geográfico y cultural inmediato en el que ejerció su actividad docente e investigativa, e incluso encuentra ecos en los debates que se dan en la época actual acerca de ciertas cuestiones candentes en el estudio de las lenguas y culturas en América. En este sentido, Lenz resulta ser un caso ejemplar del intelectual (¿orgánico?) inmerso en la creación, difusión y renovación de paradigmas en un contexto sociopolítico en transformación y encaminado hacia la modernidad. Las incursiones de Lenz en la esfera pública chilena de fines del XIX y comienzos del XX incidieron de modo significativo en la constitución de las representaciones acerca de la relación entre lengua(s), cultura(s) y nación(es) en el Chile de entonces, en pleno proceso de modernización. La labor de Lenz, sin embargo, se desarrolló en un entorno donde el estudio del lenguaje y la planificación lingüística ya gozaban de amplio arraigo en el marco de los intereses de la esfera pública, aunque habían tenido una naturaleza y propósitos distintos de los de la nueva ciencia lingüística promovida por el alemán.

Heller y McElhinny (2017, cap. 4) sostienen que la unificación del mercado lingüístico en los países europeos sirvió de base para el funcionamiento del capitalismo y la democracia liberal que organizan los Estados modernos. Aún más, argumentan que la construcción y naturalización de una representación del lenguaje según la cual este es un recurso neutro, homogéneo y gobernado por reglas, igualmente accesible a todos los ciudadanos mediante la educación (una supuesta "norma culta" estándar), sirve para el enmascaramiento del acceso desigual a la riqueza, transgresión inconfesable pero fundamental de la promesa capitalista. Esta representación sirve como un terreno fructífero para la legitimación del poder de la clase dominante al mismo tiempo que construye un espacio compartido que facilita la sensación de pertenencia de los ciudadanos al Estado-nación. 
En el caso de Chile, los fundamentos ideológicos del rol político del lenguaje pueden rastrearse hasta la figura de Andrés Bello, artífice de la ideología lingüística hegemónica de la intelectualidad chilena durante todo el siglo XIX: la del orden de la lengua, que sigue vigente en lo esencial (Rojas, Avilés y Villarroel, en prensa). La unificación del mercado lingüístico calzaba perfectamente con la obsesión por el orden, central tanto en el imaginario de Bello (Jaksić 2010) como en el de las élites gobernantes chilenas (Stuven 2000). Para Bello, el orden de la lengua (española, se entiende, por ser esta la propia de los criollos que llevaron adelante la independencia y cuya posición privilegiada debía ser legitimada), no podía sino darlo una norma estándar academicista con firmes raíces en la tradición hispánica, que ponía como conducta ejemplar el dialecto castellano, la lengua de los cultos y el código escrito (Moré 2014), y que al mismo tiempo contenía dentro de sí la posibilidad de albergar cambios que contribuyeran al progreso de las naciones americanas, siempre que estos cambios se mantuvieran dentro de los cauces de la tradición (Rojas 2017).

Al mismo tiempo, la representación ideológica originada en Bello ponía a los grupos subalternos ("vulgo", "populacho" o "plebe", prefirió llamarlos), hablantes de castellano "subestándar", como antiejemplo de conducta, y como responsables de los "vicios", "pecados" y "delitos" idiomáticos que amenazaban con fragmentar la lengua y así levantar "estorbos a la difusión de las luces, a la ejecución de las leyes, a la administración del Estado, a la unidad nacional" (Bello [1847] 2013: 43). Bello tenía conciencia de estarse enfrentando a un problema no solo de planificación lingüística, sino primero que nada de regulación y naturalización del orden social, político y económico: "En las lenguas como en la política, es indispensable que haya un cuerpo de sabios, que así dicte las leyes convenientes a sus necesidades; como las del habla en que ha de expresarlas; y no sería menos ridículo confiar al pueblo la decisión de sus leyes, que autorizarle en la formación del idioma" (Bello [1842] 2013: 100).

Los estudios del lenguaje en Chile hasta la llegada de Lenz, en aplastante mayoría, responden a esta necesidad percibida de construir y preservar esa norma estándar (castellanizante y academizante) que garantizaría la preservación de los privilegios de la clase dominante. Es, en consecuencia, una lingüística normativa, prescriptiva, orientada a disciplinar a los ciudadanos en el uso correcto de la lengua unificada del Estado, que tenía bastantes diferencias respecto de los hábitos lingüísticos vernáculos de la mayor parte de la población chilena. Un ejemplo muy representativo de ello es la tradición de los diccionarios de chilenismos (Rojas 2010). Gracias a la influencia de que gozó Bello en la conformación de distintas instituciones reguladoras de la ciudadanía, entre ellas el sistema educativo, su ideología 
lingüística tuvo condiciones óptimas para convertirse ya en el momento de su muerte (1865) en una especie de sentido común incuestionable de la comunidad hispanohablante chilena, una suerte de habitus bellista, conformándose en consecuencia una hegemonía de efectos duraderos y una comunidad fuertemente cohesionada de epígonos de Bello que saturaron las intervenciones públicas en el espacio del lenguaje durante todo el resto del siglo XIX.

Es a este contexto al que se enfrenta Lenz cuando llega a Chile e introduce una fractura tanto en la manera de entender el estudio del lenguaje como en el modo de imaginar la relación entre lenguas, culturas y naciones. Y es que el sentido común de Lenz acerca del objetivo de los estudios lingüísticos, correspondiente a aquel de la disciplina ya establecida como ciencia del lenguaje en Alemania, colisionó frontalmente con el sentido común de la hegemonía bellista en Chile, así como con el del hispanismo academicista a nivel internacional (como muestra Bernaschina 2013, a propósito de la recepción de las ideas de Lenz en la Escuela Filológica Española encabezada por Menéndez Pidal). Un ejemplo prístino de este conflicto es la crítica del sacerdote e hispanista chileno Manuel Antonio Román al Diccionario etimológico de Lenz (cf. Pavez 2015: 130-132):

Hasta ahora todos habíamos creido que la enseñanza de la gramática era para hablar y escribir correctamente, como la gente educada; pero ahora el Dr. Lenz nos dice que estábamos profundamente equivocados y que la gramática, por lo menos en Chile, es para hablar y escribir 'la lengua huasa'. (cit. en Lenz, s.f.)

Por esta razón hemos creído y creemos que todo el que se dedique al estudio del lenguaje tiene la noble misión de encauzarlo en las leyes de la gramática, desbastándolo, puliéndolo y limpiándolo según las exigencias de la civilización y de la simple educación. Si así no fuera, la ciencia del lenguaje no sería ciencia sino mero inventario de palabras, desde la que estropea con su mala pronunciación el último patán, hasta las que profieren con cínica desvergüenza el borracho en la taberna y el frecuentador de burdeles. (cit. en Lenz, s.f.: )

A esto, Lenz contesta lo que sigue, revelando los horizontes completamente distintos que tenían, por un lado, los especialistas científicos de la época (representados por Lenz) y, por el otro, los cultores del normativismo (representados por Román):

Esta definición del estudio del lenguaje (digamos de la lingüística) es tan correcta como si se dijera que la botánica tiene por objeto producir hermosas flores, peras dulces y papas grandes. La confusion entre 
retórica y lingüística es a lo menos falta tan grave como si se declarara que horticultura y botánica son una misma cosa. La mala pronunciación del último patan puede tener para la lingüística el mismo interés que un cultivo de bacilos de la peste bubónica para la biología y la medicina. (Lenz, s.f.).

Este tipo de comparaciones pueden encontrarse de manera recurrente a lo largo de la obra de Lenz, así como la insistencia en demarcar el terreno propio del especialista de una disciplina establecida con la legitimidad del saber científico y aquellas prácticas que esa misma afirmación había venido a relegar fuera de ese ámbito (Ennis 2012: 204-205). En cualquier caso, la colisión tuvo peores resultados para el alemán que para el bellismo, que persiste hoy prácticamente intacto, principalmente al alero de instituciones fuertemente comprometidas con el hispanismo y con gran influencia en el mundo universitario, como la Academia Chilena de la Lengua.

Más allá de las polémicas con Eduardo de la Barra o Manuel Antonio Román (v. Velleman 2007, Altschul 2011 y Pavez 2015: 133-144), el gran antagonista de Lenz fue el fantasma de Bello y del orden social que este contribuyó a naturalizar: la inercia aplastante del "peso de la noche" portaliano, del imaginario clasista y racista fuertemente arraigado en diversos sitios institucionales de la nación. Desde mediados de la década que acaba de terminar, varios investigadores han venido insistiendo acertadamente en la necesidad de reivindicar la radicalidad glotopolítica de las intervenciones de Lenz:

Siguiendo con atención las propuestas de Lenz, resulta ostensible que el foco de atención y preocupaciones de los estudios filológicos cambia. El objetivo fundamental de una filología como ésta no es entonces la recomposición de la unidad esencial de una lengua, su sistema inmutable, sino precisamente su heterogeneidad: la indagación de aquellos puntos de articulación con formas de expresión provenientes de otros estratos sociales y comunidades étnicas, lo que implica una investigación radicalmente distinta, en la que es imprescindible aceptar una historia de la lengua diferente y desear contarla en sus propios términos. (Bernaschina 2013: 132)

Lo escandaloso de la posición de Lenz reside -a mi modo de ver- en la forma en que le asigna un rol activo en la formación del español de Chile a la población de las capas sociales bajas -según él, en su mayoría de origen indígena- y en consecuencia en la cuestión de la formación nacional. La diferencia de Chile consistiría entonces en el hecho de que las masas populares y las clases dirigentes hablasen el mismo idioma y que así se hiciera palpable la comunidad imaginada. Sólo que esta 
comunidad imaginada no se restringe a la población letrada que comparte la circulación de la imprenta, sino que integra a toda la población en un cuerpo lengua-nación. [...] A diferencia de esta visión, los proyectos lingüístico-nacionales anteriores partieron, como bien demuestra González-Stephan (1995) en su artículo sobre las disciplinas escriturarias, de las élites criollas que impusieron estas disciplinas por medio de su lengua, sus leyes y sus modales de comportamiento y que vieron a las clases bajas sólo como objeto de procedimientos correccionales o de exclusión social. (Bachmann 2014: 259-260)

Se trata de una relación con el pasado, con la nación y con el "valor de antigüedad" que la élite chilena no comprendía, y de lo cual desesperaba Lenz. Como tuvo ocasión de señalar en varias oportunidades y con altos costos personales, la élite chilena no tenía vocación de integración nacional, ni la voluntad de participar en un "espíritu" común con el pueblo chileno. Este clasismo que obstaculizaba la formación de la nación chocaba permanentemente al científico alemán, cuya ciencia por racional que fuera estaba al servicio de un afecto nacional. (Pavez 2015: 164)

La complejidad glotopolítica de la obra de Lenz y la relevancia social de las interrogantes que lo ocuparon en Chile, precisamente, fue una de nuestras motivaciones para articular el presente proyecto editorial, con el cual nos proponemos concitar no solo el interés de lingüistas y antropólogos, habitualmente conocedores de la figura de Lenz, sino de todas las personas interesadas en las políticas de la cultura y del conocimiento en América Latina.

Sin embargo, reconocer que Lenz se posicionó a contrapelo de las ideas hegemónicas del hispanismo en Chile no puede llevar por necesidad a concluir que los efectos glotopolíticos de su obra fueran contrarios a los propósitos de la hegemonía bellista. Después de todo, como advierte Joseph Errington respecto de la lingüística colonial europea, "linguists' work had meanings and uses which outstripped their own purposes and understandings" (Errington 2008: 12). En un estudio reciente, Cáceres, Ortiz y Rojas (2020) argumentan que el Diccionario etimológico de Lenz, lejos de ser un espaldarazo a la vitalidad de la lengua mapuche, puso en circulación y contribuyó a legitimar (desde el lugar lenziano del científico respaldado por el Estado) una representación del mapudungun como un futuro fósil, museificable a través del estudio de su inscripción intersticial en el castellano chileno, y del mapuche como un sujeto que debía ser asimilado a la nación chilena, convirtiéndose en un sujeto monolingüe en un castellano, eso sí, marcado por las huellas del contacto. De esta manera, la obra de Lenz pudo ser útil para los intereses del colonialismo republicano y entró en una 
sorprendente sinergia con las representaciones provenientes del hispanismo bellista (cf. Rojas 2020).

Más allá de la responsabilidad que se pueda achacar a Lenz respecto de los efectos glotopolíticos de su trabajo, creemos conveniente adoptar una distancia crítica al momento de estudiar la obra del lingüista alemán, como es deseable hacerlo al abordar la obra de cualquier figura intelectual, por simpática que pueda parecernos y por mucho que podamos empatizar con sus supuestos epistemológicos y políticos. Así, la labor de estudiar a Lenz podrá ser, más que una hagiografía vana (inclinada a una especie de beatificación de su figura), un ejercicio de comprensión histórica del que sacar lecciones relevantes para los procesos de construcción de conocimientos en que nos encontramos inmersos en la actualidad.

\section{LA TRAYECTORIA INTELECTUAL Y BIOGRÁFICA DE LENZ}

Rodolfo Lenz nació, con el nombre de Rudolf, un 10 de septiembre de 1863 , en uno de los centros más prestigiosos de la lingüística decimonónica, la ciudad de Halle an der Saale -la ciudad, sin ir más lejos, hacia la cual peregrinaría quince años más tarde Rufino José Cuervo para entrevistarse con August Friedrich Pott, quien contribuiría en una breve entrevista en latín a imprimir un giro decisivo en la obra del bogotano, y con ello a la suerte de las ciencias del lenguaje en estas latitudes (cf. Pfänder 2006, Pfänder y Ennis 2015). Sin embargo, no puede pensarse que haya sido mucho más que la casualidad lo que desde su nacimiento vinculó a Rudolf Lenz con la tradición más sólida de la lingüística decimonónica. Su padre, funcionario de correos, había sido asignado allí por un tiempo, y la familia abandonaría la ciudad pocos años después, aún durante su infancia. Fue en Bonn, en la universidad y la cátedra pionera de la Romanistik donde, a las 11 horas de un 11 de agosto de 1886, a los 23 años, entregó una tesis sobre fisiología e historia de las palatales (Physiologie und Geschichte der Palatalen), publicada poco más tarde por la editorial Bertelsmann de Gütersloh, y dirigida por Wendelin Foerster, catedrático sucesor del mismísimo Friedrich Diez, a quien - de acuerdo con una tradición que va de Gaston Paris a Carlo Tagliavini al menos- el mismo Goethe habría puesto en el camino pionero de la filología romance, que conduciría a su célebre Etymologisches Wörterbuch der romanischen Sprachen (Bonn, 1854; cf. Tagliavini 1982: 10).

La tesis del joven Lenz se proponía realizar un aporte técnico y teórico a la lingüística histórica, a tono con la fe de la corriente dominante de los 
Junggrammatiker en el poder explicativo de las leyes fonéticas, aunque privilegiando una descripción fundada en la observación empírica de los rasgos fisiológicos de la producción de los sonidos que rápidamente pondría límites y reparos a esos presupuestos, apuntando así a una actualización teórica a partir de la revisión de los presupuestos empíricos de las Lautgesetze que, como habían establecido poco antes Osthoff y Brugmann (1878), debían explicar las reglas del cambio lingüístico.

El Doctor Rodolfo Lenz, luego de haber defendido con éxito su tesis, recibió del gobierno chileno la propuesta de incorporarse al plantel docente del recientemente fundado Instituto Pedagógico de Santiago. El 4 de noviembre de 1889 firmaba en Berlín, ante el Ministro Plenipotenciario de Chile en Alemania, Domingo Gana, un contrato por seis años como profesor de los idiomas inglés, francés e italiano. A pesar de la aparente limitación que el objeto del contrato oponía en principio a la carrera de Lenz como lingüista profesional, es sabido que desde su llegada a Chile prosiguió y profundizó su trabajo y formación como investigador, abocándose al estudio del español de Chile y las diversas variedades del mapudungun. Si bien puede observarse, tanto en sus publicaciones como en su correspondencia personal, un reiterado lamento por la dificultad, y muchas veces imposibilidad que su nueva situación periférica con respecto a los centros de producción del saber lingüístico y filológico suponía para la obtención de los materiales y el cultivo del contacto con los colegas que podía permitirle estar actualizado, el tiempo y la distancia, en buena medida, continuarían jugando a su favor, ofreciéndole la oportunidad de acceder al conocimiento de una serie combinada de objetos diversos, vacantes aún en la cartografía que la lingüística moderna trataba de establecer en sincronía y diacronía alrededor del mundo, terra incognita de la convivencia americana del español colonial y las lenguas indígenas, y al mismo tiempo de hacerlo desde la perspectiva del lingüista, ya no como alguno de sus antecesores nativos, por apropiación de su discurso, sino con la legitimidad y solidez de conocimientos que le otorgaba su formación, y con la posibilidad, al acceder a la novedad de este objeto, al proveer de materiales que enriquecieran el acervo de la investigación metropolitana, de mantenerse, si no reconocido, al menos reconocible en los centros de producción y legitimación del saber especializado.

Es difícil calibrar verdaderamente la novedad de esta escucha puesta en el habla cotidiana de todas las clases, en especial -y de acuerdo con la impronta romántica que, aún después de los Junggrammatiker, caracterizaba la formación del lingüista- las más postergadas en América. El descubrimiento de la historia y presente de contacto, conflicto e intercambio entre una lengua y cultura indígena americana de características tan especiales como la mapuche y el español de la sociedad criolla que había asistido a la Gramática 
de Bello resultarán decisivas en la afirmación de su carrera como lingüista profesional, a pesar de que esas tareas, oficialmente, no fueran aquellas objeto de su empleo. Toda la serie de trabajos publicados mayoritariamente en los Anales de la Universidad de Chile, que tiene un punto de partida en aquel sobre "Lingüística americana" firmado junto a Barros Arana su tónica, y se sostiene luego en la serie de Estudios araucanos, encuentra su respaldo en la recepción en el sistema científico de procedencia del resultado de esas mismas investigaciones en revistas prestigiosas como la de Gröber, o el conocido folleto de 1902, Die indianische Elemente im Chilenospanischen, publicado por la editorial Niemeyer, entonces radicada en su natal ciudad de Halle.

Uno de los síntomas más claros de su éxito en este afán de visibilidad, más allá de los reiteradamente registrados en la propia producción, se encuentra en el modo en el cual Wilhelm Meyer-Lübke introduce su referencia en su Einführung in das Studium der romanischen Sprachwissenschaft, a tal punto que en la reedición en 1926 de la traducción castellana de 1914 (que aparece en la misma colección que albergara La oración y sus partes), bajo el título de Lingüística románica, Américo Castro debe dedicar media página de nota al pie a conjurar la reproducción de las hipótesis de Lenz por el profesor austriaco (Meyer-Lübke 1926: 332). La célebre tesis sustratista -según la cual el español de Chile obtendría sus rasgos fonéticos distintivos del contacto con el mapudungun- se había convertido en su seña de identidad más visible e inquietante, aquella cuya refutación Amado Alonso llevaría después de su muerte incluso a la misma edición de las obras de su autor (Ennis 2012: 196-203).

Meyer-Lübke, representante asimismo de la corriente dominante en el final del XIX, la de los Junggrammatiker, otorgaba así a Lenz un reconocimiento que ponía de relieve su rol pionero no solo en América, sino también con respecto a España. Sin embargo, aún en su siempre subrayada posición periférica, Lenz obtendría el reconocimiento asimismo del polo opuesto del campo científico en el estudio del lenguaje, y la visibilidad así para la novedad de su objeto. En una reseña de dos trabajos de Fredrik Wulff, Hugo Schuchardt -que especialmente con su polémico trabajo de 1885, Über die Lautgesetze. Gegen die Junggrammatiker, se había convertido en uno de los más sólidos críticos de la escuela neogramática- hacía una mención y comentario mesuradamente elogiosos y no carentes de crítica de la tesis doctoral de Lenz, donde indicaba justamente que este había mostrado un camino en su tesis para resolver los problemas que encontraba abiertos en la representación de la fonética de las lenguas romance en Meyer-Lübke, y esto a través de un conocimiento especializado y empírico (Schuchardt 1892: 244). La crítica apuntaba sobre todo a las limitaciones que imponía a su 
desarrollo el haberse restringido a las lenguas románicas. Como lo muestran Sousa y Mücke (2016; cf. también Mücke y Sousa en este volumen), Lenz tomará nota de esta mención, y enviará una carta al profesor de Graz, en la que agradece la consideración prestada y responde a las críticas a partir de las limitaciones previas, impuestas por su falta de especialización en lenguas fuera del alemán y las romance, limitaciones que ahora en Chile puede superar $-y$ lo habría logrado con su famosa hipótesis indigenista. Dice un pasaje de la carta del 31 de julio de 1892:

Como sostengo, he demostrado con seguridad que casi el completo desarrollo de la fonética chilena se funda en la influencia del araucano, un hecho de historia lingüística como no ha podido constatarse nunca hasta ahora con tal claridad.

En este sentido, Lenz constituye un caso excepcional -no único, por supuesto, pero sí fuera de la regla habitual (cf. Pavez 2015: 61)- no va a la periferia y vuelve al centro, no recoge la información que le traen otros, sino que, al mismo tiempo que configura su objeto, procura generar las condiciones de su legibilidad in situ, produce el saber allí donde hace el trabajo de campo. Así lo destaca en sus trabajos, subrayando su condición de conocedor directo de la materia investigada, y privilegiado a la vez con respecto a sus predecesores (sobre todo la lingüística misionera) por su condición de especialista formado en el marco epistemológico e institucional de la lingüística moderna.

Las décadas posteriores añaden a sus investigaciones un mayor empeño en la intervención del especialista en el espacio y las políticas públicas sobre la lengua, tanto en lo relativo a la enseñanza de la gramática, lenguas extranjeras y en la patrimonialización de las lenguas americanas, sobre todo el mapudungun.

De tal modo, aún en la proclamada distancia chilena, Lenz podía mantener su interés científico abierto a las posibilidades de la lingüística metropolitana y sus alrededores, lo cual se hará aún más claramente manifiesto en las dos monografías que, junto al Diccionario, completan el esquema más básico de su trayectoria: La oración y sus partes (1919) -quinto volumen de las "Publicaciones de la Revista de Filología Española" de Menéndez Pidal-, donde hay una fuerte apuesta por el enfoque de Wundt, por un lado, y El papiamento (1928), publicado en los Anales, en diálogo con Schuchardt y nuevamente con una apuesta decidida por tesis aún novedosas, como por ejemplo las de Otto Jespersen (1922).

Su obra fundamental, aquella a la que apunta casi todo su trabajo y que ocupa buena parte del diálogo con sus corresponsales, queda inconclusa (como el Diccionario de construcción y régimen de Cuervo, probablemente esa sea su naturaleza), y será publicada como libro en 1987 por Mario 
Ferreccio, el Diccionario Etimológico de las voces chilenas derivadas de lenguas indígenas americanas. Es en el prólogo a esa edición que Ferreccio (1979: 13) apunta una de las ponderaciones más extremas del lingüista alemán, interesante sin embargo para pensarla a la luz de la lógica de marginación y recentramiento que atraviesa su carrera: "Es difícil escatimar elogios a Rodolfo Lenz, es difícil exagerarlos. Distintos factores incidentales han conducido a no proclamarse lo que en realidad es: uno de los fundadores centrales de la Filología Románica por la incorporación de un campo nuevo a esta disciplina".

Lenz nunca deja de tener esa posición doblemente marginal: al margen del medio de procedencia, al margen de la cultura de acogida. Artífice de la legitimación científica de un proyecto colonial, no deja de ser cuestionado desde el comienzo por las élites locales (cf. Poblete 2002, Altschul 2012, Pavez 2015), con las que mantiene una relación siempre llena de suspicacias. Ese relativo aislamiento se compensa con la construcción de una densa red de contactos y colaboraciones dentro del continente y entre continentes. El abundante epistolario que registra el Fondo Rodolfo Lenz en la Universidad Metropolitana de Ciencias de la Educación en Santiago de Chile enseña los trazos aún difusos de un denso y persistente entramado entre América y Europa a través del cual Lenz, procurando sostener el diálogo, realizó una contribución decisiva a sus mismas condiciones de posibilidad. Este dosier contiene contribuciones acerca del intercambio con Schuchardt y Lehmann-Nitsche, dos de los más interesantes probablemente, aunque hay mucho más por explorar aún.

La excepcionalidad de la trayectoria de Lenz ofrece un punto de mira privilegiado para observar la historia de la filología americana con todos sus rasgos, sobre todo sus intrincadas discontinuidades. Desde la cuna de la filología románica hasta sus confines, tanto geográficos como lingüísticos, entre el español de Chile y el papiamento, la enseñanza del francés, las políticas del español y el estudio del mapudungun o "araucano", entramando y entramado en redes intelectuales de diversa densidad entre Europa y América Latina, ofrece una mirada cuyo sesgo resulta tan interesante como su lucidez. Al igual que Cuervo, y en parte contemporáneamente, viene a llenar un hueco autoproclamado, y de otro modo: mientras Cuervo, para terminar de convertirse en el primer lingüista americano, se traslada a París, Lenz, ya formado como lingüista, se radica en Chile. Su trabajo se desarrolla sobre un fondo que él mismo se ocupa de montar recurrentemente, y le permite ensayar el tono de una predicación secular, la de la ciencia lingüística, dirigida siempre a un público postergado en el tiempo y el espacio. La primera nota a pie de página en el "Prólogo" al Diccionario remite a la "bibliografía crítica" que lo acompaña, "en la cual espongo mi opinion con entera franqueza, que 
estrañará quizás a mis lectores americanos; estoi seguro que los filólogos europeos me la agradecerán, i esto para mí es decisivo" (Lenz [1905-1910] 1979: 8). Allí se destaca una vez más el doble obstáculo a superar en los estudios lingüísticos americanos -la labor de los misioneros, que al pasar por la criba del latín y de su fin proselitista distorsiona el registro de las lenguas americanas, por un lado, y por el otro la pobreza y el mal entendido casticismo de la mayor parte de los trabajos que encuentra en la América contemporánea acerca del español allí hablado- así como el destinatario último de esa labor: un público de pares, solo asequible en Europa.

El perfil de Lenz va adquiriendo un contorno claro así a través de una especialización técnica y teórica inicial (la lingüística románica, la fonética acústica y articulatoria) que encuentra en la singularidad de su campo de acción y aplicación sus rasgos más controvertidos y perdurables. Como escribirá en unas líneas retrospectivas de 1924:

La primera tarea científica a que dediqué mis horas libres ha sido el estudio del dialecto vulgar chileno. En Enero de 1891 mandé el primero de mis Chilenische Studien a la revista alemana "Phonetische Studien", editada en Marburg por W. Viëtor, porque, si lo hubiera publicado en Chile, los "literatos" se habrían reído del gringo loco que tratara los "vicios de lenguaje de la plebe ignorante" como materia digna de trabajos científicos.

Sin embargo, aseverará, sin modestia alguna, "[h]oy han cambiado estas cosas. Desde los últimos años del siglo pasado nació en Chile la filología española y poco después también en España Ramón Menéndez Pidal comenzó a formar su escuela brillante de filólogos. Tan luego como tenga tiempo pienso publicar una reseña sobre el dialecto popular de Chile, que ya encontrará lectores interesados y preparados" (Lenz 1924: 10-11). Ese optimismo hace posible que una obra como El papiamento se publique en Chile, y encuentra su ratificación -y sus límites- en la repercusión y polémica que despierta, tanto en Chile (v. Emeth 1940: 369-373) como más allá de sus fronteras (Costa Álvarez 1928, pero también Nykl 1928).

Rodolfo Lenz muere en Santiago de Chile un 7 de septiembre de 1938. En el homenaje que la Universidad de Chile brinda a su memoria, Amado Alonso, director desde 1927 del Instituto de Filología de la Universidad de Buenos Aires, describirá al "Doctor Rodolfo Lenz" como "uno de los pocos hombres" que, "por los años ochenta del siglo pasado [...] convirtieron la observación de las pronunciaciones en una ciencia" (Alonso 1938: 11). En la misma exposición, anunciaba la preparación del tomo VI de la Biblioteca de Dialectología Hispanoamericana, titulado El español en Chile, conteniendo trabajos de Lenz (junto a una porción menor de Bello y 
Rodolfo Oroz), traducidos por el propio Alonso y Raimundo Lida, entre los que se contaban los Chilenische Studien, publicados por Lenz en Halle en 1892-93, "sin duda [...] la primera descripción fonética satisfactoria de un dialecto hispánico" (Alonso 1938:13). Al volumen anunciado por Alonso se añadirían, finalmente, cuatro apéndices, dos de los cuales son los artículos del mismo Alonso dedicados a disputar la tesis sustratista de Lenz sobre el español chileno. Este dato no es irrelevante, por cuanto, tal como recuerdan G. Knauer y M. Kaluza (1998) -siguiendo en este punto tanto a Petursson (1989) como a Zimmermann (1995)-, conocemos las tesis de Lenz sobre todo a través de las críticas y refutación de Alonso ${ }^{5}$. Este último reconocimiento y reconvención ilustran bien la peculiar parábola que describe el trabajo de Lenz. No hay una continuidad clara, medio siglo de labor en Chile no parece dejar una secuela evidente y solo mucho tiempo después comienza a revisitarse su obra, que recién hoy, giro archivístico mediante, comienza a recibir todo el interés que merece.

\section{EL RETORNO DE LENZ}

Al organizar el presente dosier (originado en el Coloquio Rodolfo Lenz celebrado en octubre de 2016 en la Facultad de Filosofía y Humanidades de la Universidad de Chile), nos propusimos, sin ánimo hagiográfico, destacar la relevancia y actualidad de Lenz como figura intelectual representativa de las dinámicas de conformación de las disciplinas relativas a las lenguas y las culturas en el marco de la consolidación de los Estados nacionales latinoamericanos en los umbrales del siglo XX, y contribuir a revitalizar y estimular su estudio, y sobre todo el de las interrogantes que cautivaron su

\footnotetext{
5 Así puede comprobarse, en una semblanza rayana en lo hagiográfico como la de Rabanales, cómo se da por supuesto el error de Lenz, a pesar del cual puede de todas formas valorarse su obra: "Es curioso que a veces una idea falsa pueda producir efectos positivos. Tal vez Bello no habría escrito nunca su gramática magistral si no hubiera creído que con ella contribuía a evitar la fragmentación del español en América, como había ocurrido en Europa con el latín. Del mismo modo es posible que el maestro alemán no hubiera llegado a ser el primer araucanista verdaderamente científico si no hubiera dado por hecho -de acuerdo con las ideas sustrativas adquiridas durante su formación académica- una influencia decisiva del araucano o mapuche en nuestra manera, sobre todo inculta, de pronunciar el español" (Rabanales 2002: 68).
} 
interés, que gozan de una indiscutible relevancia para el momento actual de la historia.

No pretendemos ignorar que la figura y obra de Lenz han concitado ya bastante atención: prueba de ello son, por nombrar solo algunos ejemplos recientes, las indagaciones desde la historia intelectual y en particular de la historiografía de las ciencias del lenguaje ${ }^{6}$. Tampoco desconocemos que ha habido aproximaciones de gran lucidez acerca de las aristas políticas de la obra de Lenz (sobresale entre ellas el trabajo mencionado de Jorge Pavez). La principal ambición de originalidad de nuestro proyecto fue reunir por primera vez un conjunto de estudios inéditos escritos por especialistas situados en distintos enfoques disciplinares relevantes para entender la obra de Lenz (la lingüística de contacto, la glotopolítica histórica y la historiografía intelectual), con el propósito de destacar la relevancia actual de los problemas lenzianos.

Un primer grupo de trabajos de este dosier se ocupa de revisitar y reivindicar la tesis sustratista de Lenz respecto del castellano chileno, planteada primero en Lenz ([1892-1893] 1940) y desarrollada con detalle en Lenz (1893 [1940]), cuando ya deja de ser una sospecha y se convierte en "certidumbre personal" para el investigador alemán. Precisamente esta tesis era la parte de su trabajo que Lenz consideraba de mayor interés para la romanística, por los paralelos que podían establecerse entre el contacto lingüístico acaecido en Chile y aquel que había tenido lugar en Europa con la expansión del latín, y además por la peculiaridad de que la lengua de sustrato en el caso chileno todavía se hablaba, lo cual facilitaba la comprobación de hipótesis. Los reparos de Amado Alonso señalaban específicamente que no podía descartarse la posibilidad de una influencia de sustrato o adstrato mapuche "en las melodías y en los juegos rítmicos" del castellano chileno (Alonso 1940: 289), pero rechazaba de plano tal influencia "en el sistema fonético" (ibid.).

En la presente sección monográfica, Scott Sadowsky, a la vez que cuestiona la solvencia de varios de los reparos de Alonso a Lenz en lo que respecta a las consonantes (los que considera, además de equivocados, ideológicamente sesgados), introduce una nueva arista del problema: la fonética del sistema vocálico del castellano chileno, en la que concluye

\footnotetext{
6 Álvarez Martínez 1997; Bernaschina 2013, 2016; Cáceres y Rojas 2019; Cáceres, Ortiz y Rojas 2020; Chávez 2011; Concha 2019; Contreras 1994; Ennis 2012, 2016, 2020; Gómez Asencio 2016; Gómez Asencio y Rojas 2019; Knauer 1993; Knauer y Kaluza 1998; Payas 2015; Pavez 2015; Rojas 2011; Sánchez 2013; Soto 2016; Valdez y Jansen 2019; Velleman 2008, entre otros.
} 
que efectivamente existe influencia del mapudungun. Brandon Rogers, asimismo, es el primero que se ocupa de estudiar el problema de la entonación del castellano chileno en relación con la tesis sustratista de Lenz. Este investigador concluye que ciertas mesetas entonacionales que tienen por función extender el foco discursivo pueden entenderse como un aporte del mapudungun a la fonología del castellano chileno. Finalmente, Felipe Hasler, Aldo Olate y Guillermo Soto hacen una revisión de fenómenos fonológicos, morfosintácticos y pragmáticos del castellano chileno en los cuales puede identificarse la huella del contacto con el mapudungun, estableciendo la vigencia de la obra de Lenz en relación con cuestiones fundamentales de la lingüística de contacto actual. En síntesis, este grupo de trabajos dan cuenta de cómo los avances teóricos y metodológicos de la lingüística, y específicamente de la lingüística de contacto, posibilitan revisitar los postulados de Lenz y considerarlos como hipótesis válidas para emprender nuevas investigaciones hoy.

El segundo grupo de trabajos se ocupa de revisitar la figura y la obra de Lenz desde la perspectiva de la historiografía lingüística con inclinación glotopolítica afín a lo que Valdez (2015: 87) llama historiografía crítica de la lingüistica), poniendo de relieve un aspecto fundamental para comprender la inscripción de la labor lenziana en su contexto: el de las redes intelectuales en torno a las cuales se desarrolló su trabajo. Estas contribuciones permiten pensar la actividad de Lenz situándola en el marco del estudio crítico de "los modos de circulación y transferencia del conocimiento científico" (Salto y Chicote 2020: 7), tanto dentro de su país de acogida como en relación con el centro hegemónico de producción de conocimiento en su época, Europa. Es muy relevante, para dimensionar la importancia del estudio de esta arista, recordar que Lenz siempre buscó legitimidad en el reconocimiento que los grandes nombres y revistas de la ciencia lingüística europea pudieran otorgar a sus trabajos, como se puede ver claramente en el caso de las reseñas del Diccionario etimológico (Cáceres, Ortiz y Rojas 2020: 23). Asimismo, Lenz trabajó activamente en el levantamiento de redes de colaboradores o "informantes" dentro del país (Pavez 2015: 96 y ss.), los que le facilitaban materiales para sus estudios, y, además, en el caso de sus colaboradores mapuche, daban el valor agregado de la autenticidad.

El artículo de Johannes Mücke y Silvio Moreira de Sousa incluido en el presente volumen exhibe y estudia el intercambio epistolar entre Lenz y el romanista Hugo Schuchardt, figura central en el surgimiento de la criollística en Europa. Junto con contextualizar el corpus epistolar y caracterizar la relación académica entre ambos investigadores, el trabajo ofrece por primera vez una traducción al castellano de los documentos originales en alemán. El artículo de Claudio Soltmann, por su parte, explora los puentes que Lenz 
tendió con científicos alemanes establecidos en otros lugares de América, como ilustra el caso de Robert Lehmann-Nitsche (radicado en La Plata, Argentina), con quien compartió el interés por las tradiciones y lenguaje populares. Situándose esta vez dentro del contexto chileno, el trabajo de Fernando Ruiz Figueroa aborda la manera en que Lenz comenta y valoriza la obra autoetnográfica bilingüe del profesor mapuche Manuel Manquilef, con quien también mantenía intercambios epistolares frecuentes. Finalmente, Pablo Concha Ferreccio hace una aproximación original (tanto por su tema como por la interrogación glotopolítica del campo literario que supone) al modo en que Lenz influyó en el pensamiento del escritor criollista Mariano Latorre (quien fue estudiante de Lenz en el Instituto Pedagógico), llevándolo a una redefinición de sus coordenadas hermenéuticas y transformando las claves de interpretación hegemónicas del campo literario chileno de la primera mitad del siglo XX.

Queremos finalizar agradeciendo a las personas (además de los autores de los artículos) e instituciones que posibilitaron la realización de este dosier. El Coloquio Rodolfo Lenz 2016, en el que se originó este proyecto, no habría sido posible sin el patrocinio de la Facultad de Filosofía y Humanidades de la Universidad de Chile y el apoyo financiero del proyecto de investigación FONDECYT Regular 1150127 "Ideas lingüísticas en los debates sobre léxico y ortografía en Chile (1875-1927)" (CONICYT, Gobierno de Chile), dirigido por Darío Rojas. Entre ese momento inicial y la fecha de publicación de esta sección monográfica del Boletín de Filología, las discusiones teóricas y las indagaciones historiográficas que sustentaron la concepción de esta obra colectiva recibieron una acogida fructífera y un apoyo sostenido en el proyecto FONDECYT Regular 1170419 "Mediación lingüístico-cultural y castellanización en los ámbitos de contacto mapudungun-castellano derivados del proceso de chilenización de la Araucanía: producción lingüística y etnográfica, administración de justicia y escolarización (1880-1930)" (CONICYT, Gobierno de Chile), dirigido por la Dra. Gertrudis Payàs Puigarnau, de la Universidad Católica de Temuco; en el proyecto FFI2016-76874-P “Corpus diacrónico del español para el estudio de las actitudes lingüísticas en América y España (ss. XVIII y XIX)" (Ministerio de Economía y Competitividad, Gobierno de España), dirigido por el Dr. Manuel Rivas Zancarrón, de la Universidad de Cádiz; y en el proyecto H850 "Filología, lingüística y archivo: aportes hacia una historia política de la lengua entre Europa y América Latina (mediados del siglo XIX-comienzos del siglo XX)" (Universidad Nacional de La Plata), dirigido por Juan Antonio Ennis.

También agradecemos a las personas que de diversas maneras contribuyeron a la realización de este volumen, en especial a Natalia 
Villarroel, investigadora asistente del proyecto FONDECYT $1150127 \mathrm{y}$ colaboradora en el proceso de revisión y edición de los textos, y a María Eugenia Góngora, exdecana de la Facultad de Filosofía y Humanidades de la Universidad de Chile, facilitadora del apoyo institucional que dio empuje a la reunión académica de la que surgió el proyecto.

\section{REFERENCIAS BIBLIOGRÁFICAS}

Alonso, Amado. 1938. Rodolfo Lenz y la fonética del castellano. Homenaje a la memoria del Dr. Rodolfo Lenz, Anales de la Facultad de Filosofia y Educación de la Universidad de Chile II (1): 11-17.

1940. La interpretación araucana de Lenz para la pronunciación chilena. El español en Chile. Trabajos de Rodolfo Lenz, Andrés Bello y Rodolfo Oroz, pp. 281-289. Buenos Aires: Universidad de Buenos Aires.

Altschul, Nadia. 2012. Geographies of Philological Knowledge: Postcoloniality and the Transatlantic national epic. Chicago: University of Chicago Press.

Àlvarez Martínez, María Ángeles. 1997. Rodolfo Lenz: contribución gramatical y lexicográfica. Historiographia Lingüística 24 (1-2): 197-212.

BachmanN, Iris. 2014. Los intercambios científicos en la lingüística: desplazamientos geográficos e intelectuales. En Sandra Carreras y Katja Carrillo Zeiter (eds.), Las ciencias en la formación de las naciones americanas, pp. 249-269. Madrid/Frankfurt: Iberoamericana/Vervuert.

BELLO, ANDRÉs. [1842] 2013. Ejercicios populares de lengua castellana. En Andrés Bello, Gramática de la libertad. Textos sobre lengua y literatura, comps. Iván Jaksic, Fernando Lolas \& Alfredo Matus, pp. 99-101. Santiago: Universidad de Chile.

[1847] 2013. Prólogo de Gramática de la lengua castellana destinada al uso de los americanos. En Andrés Bello, Gramática de la libertad. Textos sobre lengua y literatura, comps. Iván Jaksic, Fernando Lolas \& Alfredo Matus, pp. 43-50. Santiago: Universidad de Chile.

Bernaschina, Vicente. 2013. Rodolfo Lenz, 150 años en disputa con el imperio de la Lengua. Universum 28(2): 117-138.

2016. Rodolfo Lenz: hacia una filología crítica americana. En Sergio Ugalde y Ottmar Ette (eds.), Politicas y estrategias de la crítica: ideología, historia y actores de los estudios literarios, pp. 119-137. Madrid/Frakfurt: Iberoamericana/Vervuert.

CÁCeres, VAlentina y Darío Rojas. 2019. Rodolfo Lenz y la reforma ortográfica chilena: ciencia, tradición y política del lenguaje. Boletín de Filología 54(1): 65-93.

Cáceres, Valentina, José Miguel Ortiz y Darío Rojas. 2020. El Diccionario etimológico de Rodolfo Lenz: una aproximación glotopolítica. Boletín de Filología 55(1): 13-49.

Chávez, Soledad. 2011. Ideas lingüísticas de Lenz en los paratextos de su Diccionario etimológico de las voces chilenas derivadas de lenguas indígenas americanas: vigencia y urgencia en el español de Chile. Lenguas Modernas 38: 83-106.

Concha Ferreccio, Pablo. 2019. Trasplantes del espíritu: folclor y lengua nacional en Rodolfo Lenz. Boletín de Filología 54(1): 119-151. 
CONTRERAS, Lidia. 1994. Las ideas ortográficas de Rodolfo Lenz. En Ortografía y grafémica, pp. 49-60. Madrid: Visor.

Costa Álvarez, Arturo. 1928. Una curiosidad lingüística: el papiamento. La Prensa, 20 de agosto de 1928.

Еметн, Oмer [Emilio Vaisse]. 1940. Estudios críticos de literatura chilena. Santiago: Nascimento.

Ennis, Juan Antonio. 2012. Rudolf Lenz en la encrucijada criolla. Signo y Seña 22: 181-214. 2016. Rodolfo Lenz: economías de la lengua y políticas de la lingüística. Boletín de Filología 51(1): 117-145.

2020. Intelectuales alemanes en la América remota: observaciones sobre la correspondencia entre Rudolf Lenz y Robert Lehmann-Nitsche (1898-1928). Revista Internacional de Lingüistica Iberoamericana 35: 15-30.

ERrington, JosePh. 2008. Linguistics in a colonial world. A story of language, meaning, and power. Malden: Blackwell.

Escudero, Alfonso M. 1963. Rodolfo Lenz. Thesaurus 18(2): 445-484.

Ferreccio, Mario. 1979. Presentación. En Rodolfo Lenz, Diccionario etimológico de voces chilenas derivadas de lenguas indigenas americanas. Santiago: Seminario de Filología Hispánica, Universidad de Chile.

Gómez Asencio, José J. 2016. Categorías en La oración y sus partes (1920) de Rodolfo Lenz: Clases. Boletín de Filología 51(1): 147-185.

Gómez Asencio, José J. y Darío Rojas. 2019. Rodolfo Lenz (1920) frente a la Gramática de la lengua castellana (1847) de Andrés Bello. RLA. Revista de Lingüística Teórica y Aplicada 57(1): 105-124.

Heller, Monica y Bonnie McElhinny. 2017. Language, capitalism, colonialism: toward a critical history. Toronto: University of Toronto Press.

JaKsić, Iván. 2010. Andrés Bello: la pasión por el orden. 2. ${ }^{\mathrm{a}}$ ed. Santiago: Editorial Universitaria.

JeSPersen, Отto. 1922. Language. Its Nature, Development and Origin. London: Allen \& Unwin.

Knauer, Gabriele. 1993. Filólogos alemanes en América Latina: Rodolfo Lenz y el europeísmo cultural en el discurso científico de la lingüística iberoamericana. En Hans-Otto Dill y Grabriele Knauer (eds.), Diálogo y conflicto de culturas: Estudios comparativos de procesos transculturales entre Europa y América Latina, pp. 139-152. Frankfurt: Vervuert.

Knauer, Gabriele y Martin Kaluza. 1998. Die 'indigenistische Theorie' der Phonetik im chilenischen Spanisch von Rodolfo Lenz: Ein Vorläufer der modernen Kontaktlinguistik? Philologie im Netz 3: 1-21. http://web.fu-berlin.de/phin/phin3/p3t1.htm

Lenz, Rodolfo. [1892-1893] 1940. Estudios chilenos (fonética del castellano de Chile). El español en Chile. Trabajos de Rodolfo Lenz, Andrés Bello y Rodolfo Oroz, pp. 87-258. Buenos Aires: Universidad de Buenos Aires.

[1893] 1940. Para el conocimiento del español de América. El español en Chile. Trabajos de Rodolfo Lenz, Andrés Bello y Rodolfo Oroz, pp. 211-258. Buenos Aires: Universidad de Buenos Aires.

[1905-1910] 1979. Diccionario etimológico de voces chilenas derivadas de lenguas indigenas americanas. Edición dirigida por Mario Ferreccio Podestá. Santiago: Seminario de Filología Hispánica, Universidad de Chile.

s.f. Anexo segundo al Diccionario etimológico. Edición no venal del autor.

1924. Estudio sobre los indios de Chile. Extracto de Publicaciones del Museo de Etnología y Antropología de Chile. Tomo IV, 1-2: 147-160. Biblioteca de Difusión Científica $\mathrm{N}^{\mathrm{o}} 4$, Santiago: Imprenta Cervantes. 
MeYer-LÜBKe, Wilhelm. 1926. Introducción a la lingüística románica. Versión de la tercera edición alemana, con notas y adiciones por Américo Castro. Madrid: Publicaciones de la Revista de Filología Española.

Mora, HÉctor, y Rodrigo VÁsquez. 2018. La ciencia y lo "araucano" como ideas fuerza: antropología y emergencia del "araucanismo" en Chile. En Héctor Mora y Mario Samaniego (eds.), El pueblo mapuche en la pluma de los araucanistas. Seis estudios sobre construcción de la alteridad, pp. 22-87. Santiago: Ocholibros.

Moré, Belford. 2014. Lengua y poder en la obra gramatical de Andrés Bello. Caracas: Fundación Casa Nacional de las Letras Andrés Bello.

NyKL, Alors R. 1928. Reseña: El Papiamento, la lengua criolla de Curazao: la gramática más sencilla by Rodolfo Lenz. The American Journal of Philology 49(4): 399-401.

Osthoff, Hermann y Karl Brugmann. 1878. Morphologische Untersuchungen auf dem Gebiete der indogermanischen Sprachen. Leipzig: Hirzel.

Pavez, Jorge. 2015. La lengua de Chile: Rodolfo Lenz entre chilenos y mapuches. En Laboratorios etnográficos. Los archivos de la antropología en Chile (1880-1980), pp. 67-167. Santiago: Ediciones Universidad Alberto Hurtado.

PAYAS, GERTRUDIS. 2015. Tan verídica como patriota: La pugna sobre traducción entre Rodolfo Lenz y Manuel Manquilef. CUHSO 25(2): 83-114.

Petursson, Magnus. 1989. Rodolfo Lenz y la Fonética. Actas del Octavo Seminario de la Investigación y Enseñanza de la Lingüística, pp. 3-11. Universidad de Santiago de Chile y Sociedad Chilena de Lingüística.

Pfänder, SteFan. 2006. Sprache: Variation und Wandel - Die philologische Korrespondenz Rufino José Cuervo - August Friedrich Pott 1876. En Gertrud Bense, Gerhard Meiser y Edeltraud Werner (eds.), August Friedrich Pott. Beiträge der Halleschen Tagung anlässlich des zweihundertsten Geburtstages von August Friedrich Pott (1802-1887), pp. 35-49. Frankfurt: Peter Lang.

Pfänder, Stefan y Juan EnNis. 2015. Migración sin retorno, pero con devolución: Rufino José Cuervo, August Friedrich Pott y la muerte del español. En Marco Thomas Bosshard y Andreas Gelz (eds.), Return Migration in Romance Cultures, pp. 143-169. Freiburg: Rombach.

Poblete, JuAn. 2002. Literatura chilena siglo del XIX: entre públicos lectores y figuras autorales. Santiago: Cuarto Propio.

Rabanales, Ambrosio. 2002. Rodolfo Lenz. Onomázein 7: 161-181.

ROJAS, DARío. 2010. Estandarización lingüística y pragmática del diccionario: forma y función de los 'diccionarios de provincialismos' chilenos. Boletín de Filología 45(1): 209-233.

2011. Voces usadas en Chile (1900): las cartas de Aníbal Echeverría y Reyes a Rodolfo Lenz. Onomázein 24: 349-361.

2017. Representaciones del cambio lingüístico en Chile durante el siglo XIX: ‘¿progreso o decadencia?’. Literatura y Lingüística 36: 243-262.

2020. La representación ideológica del contacto castellano-mapudungun en la lexicografía normativista chilena de fines del XIX. En Manuel Rivas y Victoriano Gaviño (eds.), Creencias y actitudes ante la lengua en la España y América de los siglos XVIII y XIX, pp. 385-401. Madrid/Frankfurt: Iberoamericana/Vervuert.

Rojas, Darío, Tania Avilés y Natalia Villarroel, en prensa. El orden de la lengua: la formación de un imaginario sobre el castellano en Chile en su primer siglo de independencia (1829-1927). En Brandon Rogers y Mauricio Figueroa (eds.), Lingüistica del castellano chileno: estudios sobre variación, innovación, contacto e identidad. Wilmington: Vernon Press.

Rojas Carrasco, Guillermo. 1940. Filología chilena. Guía bibliográfica y crítica. Santiago: Ediciones de la Universidad de Chile. 
Salto, Graciela y Gloria Chicote. 2020. Introducción. Intercambios epistolares entre lingüistas y filólogos en la primera mitad del siglo XX. Revista Internacional de Lingüística Iberoamericana 35: 7-13.

SÁncheZ, Gilberto. 2013. El Dr. Rodolfo Lenz, primer investigador científico de la lengua y cultura mapuches. Lenguas Modernas 42(2): 99-113.

Sanhueza, Carlos. 2010. Circulación de intelectuales alemanes en Chile y chilenos en Alemania (fines del siglo XIX y comienzos del siglo XX). História: Questoes \& Debates 53: 67-84.

Schuchardt, Hugo. 1885. Über die Lautgesetze. Gegen die Junggrammatiker. Berlin: Oppenheim.

Schuchardt, Hugo. 1892. [Res. de] Wulff, Fredrik, Un chapitre de phonétique avec transcription d'un texte andalou; Von der Rolle des Akzentes in der Versbildung. Literaturblatt für germanische und romanische Philologie 13: 235-246 [en línea]. Disponible en http://schuchardt.uni-graz.at/id/publication/10 (Consulta 28/05/2020).

Soto, Guillermo. 2016. Rodolfo Lenz y la enseñanza del castellano como idioma patrio en Chile. Boletín de Filología 51(1): 211-238.

Sousa, Silvio Moreira De y Johannes Mücke. 2016. Addendum to Networking and obstacles to the development of the language sciences as reflected in the correspondence of Rodolfo Lenz and Hugo Schuchardt. History and Philosophy of the Language Sciences. https:// hiphilangsci.net/2016/02/17/networking-and-the-obstacles-to-the-development-of-thelanguage-sciences/\#comment-9881

Stuven, Ana María. 2000. La seducción de un orden. Las elites y la construcción de Chile en las polémicas culturales y políticas del siglo XIX. Santiago: Ediciones de la Universidad Católica de Chile.

Subercaseaux, Bernardo. 2011. Historia de las ideas y la cultura en Chile: desde la Independencia hasta el Bicentenario. Tomo 1, volumen 1: Sociedad y cultura liberal en el siglo XIX. Santiago: Editorial Universitaria.

TAGLIAVInI, CARLO. 1982. Le origini delle lingue neolatini. Introduzione alla filologia romanza. Bolonia: Pàtron.

Valdez, Juan. 2015. En busca de la identidad. La obra de Pedro Henríquez Ureña. Buenos Aires: Katatay Ediciones.

VALDEZ, JuAn y SiLKe JANSEN. 2019. Entre la buena voluntad y la compulsión hegemónica: las implicaciones políticas del intercambio filológico latinoamericano-alemán. Boletín de Filología 54(2): 351-371.

Velleman, Barry L. 2007. Las ideas lingüísticas y pedagógicas de Eduardo de la Barra y su polémica con Lenz y Hanssen. Contextos 18: 179-193.

2008. La imagen y los ecos del lingüista profesional: la correspondencia de Rodolfo Lenz. RLA. Revista de Lingüística Teórica y Aplicada 46(1): 11-28.

ZimmermanN, KLaus. 1995. Aspectos teóricos y metodológicos de la investigación sobre el contacto de lenguas en Hispanoamérica. Klaus Zimmermann (ed.). Lenguas en contacto en Hispanoamérica. Nuevos enfoques, pp. 9-34. Frankfurt: Vervuert. 